\title{
Coleta e caracterização morfológica de populações de porongo - Lagenaria siceraria (Mol.) Standl. - Cucurbitaceae
}

ANDRE LUIS LOPES DA SILVA

Renato Aquino Záchia

DILSON ANTONNIO BISOGNIN

Jean Carlos Budke

\section{RESUMO}

Foram coletados frutos de Lagenaria siceraria (Mol.) Standl. nos municípios de Santa Maria e Jaguarí (RS) com o objetivo de caracterizar algumas raças locais utilizadas pelos produtores. Três populações de $L$. siceraria foram caracterizadas e classificadas como casco-grosso caciquense, casco-fino caciquense e casco-grosso precoce. Foi feita uma caracterização morfológica baseada em descritores de fruto maduro, visando verificar a divergência genética existente entre estas raças locais. Os dados foram submetidos a análise de agrupamento, tendo como medida de dissimilaridade a distância generalizada de Mahalanobis $\left(D^{2}\right)$ e os métodos de agrupamento utilizados foram o Método do vizinho mais próximo (Single Linkage Method) e o método de Tocher, estes métodos arranjaram as populações em dois grupos heterogêneos. As populações de porongo coletadas em Santa Maria não mostraram divergência genética sendo colocadas no mesmo grupo e a população coletada em Jaguarí foi colocada em outro grupo.

Palavras-chave : Lagenaria siceraria; Cucurbitaceae; Germoplasma; Caracterização morfológica. 


\section{SUMMARY}

Fruits of Lagenaria siceraria (Mol.) Standl. were collected in the municipal district of Santa Maria and Jaguari (RS) with the objective of characterizing some local races used by the producers. Three populations of $L$. siceraria were characterized and classified as casco-grosso caciquense, casco-fino caciquense and casco-grosso precoce. It was made a morphologic characterization based on descriptors of mature fruit, seeking to verify the existent genetic divergence among these local races. The data were submitted the grouping analysis, as dissimilarity measure was used the distance of Mahalanobis $\left(\mathrm{D}^{2}\right)$ and the used grouping methods were the "Método do vizinho mais próximo" (Single Linkage Method) and the method of Tocher, these methods obtained the populations in two heterogeneous groups. The porongo populations collected in Santa Maria didn't show genetic divergence being placed in the same group and the population collected in Jaguarí it was placed in another group.

Key-words: Lagenaria siceraria; Cucurbitaceae; Germplasm; Morphologic characterization.

\section{INTRODUÇÃO}

A cultura do porongo - Lagenaria siceraria (Mol.) Standl. - é uma atividade cujos frutos são utilizados na fabricação de cuias, recipientes usados para preparar o chimarrão, uma bebida de origem indígena. É uma atividade que se constitui na principal fonte de renda de dezenas de agricultores do Estado do Rio Grande do Sul. Esta cultura fornece matéria-prima para diversas pequenas indústrias que exportam cuias para outros estados e países vizinhos que cultivam o hábito do chimarrão (BISOGNIN \& ESTEFANEL, 1988).

A espécie $L$. siceraria possui grande variabilidade de formatos de fruto, quanto a grossura do casco, comprimento, simetria, largura e número de sementes.

Segundo ESQUINAS-ALCAZAR (1983), que apresenta escala para a comparação dos níveis de erosão e variabilidade genéticas, a saber, baixa, média, alta e muito alta; a variabilidade genética desta espécie no Brasil, é considerada alta, mas, em contrapartida, a erosão genética da mesma é muito alta.

Para o cultivo do porongo - L. siceraria, na região de Santa Maria. São utilizadas sementes oriundas de frutos selecionados, na própria lavoura comercial. Os frutos são selecionados basicamente pelo tamanho e o formato, sem nenhuma atribuição à plan- 
ta, ou seja, é uma seleção fenotípica de frutos individuais. Não se conhece a origem das populações de porongo utilizadas na região, sendo que as mesmas apresentam características fenotípicas muito similares, sugerindo origem de uma ou poucas populações e selecionadas para as diferentes necessidades ou critérios dos produtores (BISOGNIN \& MARCHEZAN,1988 apud BISOGNIN \& STORCK, 2000). A forma como as sementes são produzidas nos ajudam a compreender o manejo da variabilidade da espécie considerada.

Os elementos estudados na caracterização podem ser morfológicos, citológicos, bioquímicos ou moleculares. A maioria desses dados podem ser obtidos através de descritores, os quais constituem um atributo ou caráter observado nos acessos de um banco de germoplasma e que pode ser mensurável (HOWES, 1981).

Normalmente, os descritores estão distribuídos em listas, as quais, a princípio deveriam reunir, de uma forma ordenada e detalhada, todos os descritores individuais utilizados para uma espécie ou grupo de espécies correlacionadas (RAMOS \& QUEIROZ, 1999).

A International Plant Genetic Resources Newsletter Institute (IPGRI), publicou uma lista de descritores para Cucurbita $s p$, de autoria de Esquinas-Alcazar \& Gullick (1983), onde sugerem uma lista de descritores para várias outras cucurbitaceaes, porém sem nenhuma indicação para o gênero Lagenaria.

O objetivo deste trabalho foi analisar descritores qualitativos e quantitativos para verificar a divergência genética encontrada em frutos de porongo para posteriormente dar subsídios a trabaIhos de melhoramento genético.

\section{MATERIAL E MÉTODOS \\ Coleta de frutos}

Considerando que esta espécie é tida como sub-espontânea, as coletas limitaram-se a produtores de "cuias", foram visitados dois produtores da região de Santa Maria e um de Jaguarí. Foram analisados 16 frutos por população, totalizando 48 frutos.

\section{Caracterização Morfológica do fruto}

Os descritores utilizados para esta caracterização foram de natureza quantitativa e qualitativa. Os descritores quantitati- 
vos foram : (1) a largura do casco (epicarpo); (2) o comprimento do fruto; (3) o diâmetro maior distal do fruto; (4) o diâmetro maior proximal do fruto; (5) a largura da constrição do fruto e o (6) número de sementes do fruto.

Os descritores qualitativos avaliados foram a cavidade da região proximal do fruto (CF), que pode ser fistuloso ou cheio; os ápices (A) que podem ser agudos ou obtusos; a base (B) que pode ser convexa ou plana sendo levemente aprofundada e também o formato do fruto (FF) .

A partir da diversidade observada, sugeriu-se nomenclaturas para os formatos dos frutos encontrados nestas três populações.

Os dados obtidos pelos descritores quantitativos foram analisados pela análise de agrupamento. Foi utilizada como medida de dissimilaridade para determinar o grau de divergência entre os pares de acessos a distância generalizada de Mahalanobis $\left(D^{2}\right)$ (RAO, 1962) e para formar os grupos, foi usado o método de Tocher (CRUZ \& REGAZZI, 1994), também foi utilizado o método hierárquico do vizinho mais próximo (Single Linkage Method).

\section{RESULTADOS E DISCUSSÃO}

\section{Descritores quantitativos}

Verificou-se pela análise de agrupamento a formação de dois grupos heterogêneos. No primeiro grupo foi colocado as duas populações provenientes de Santa Maria, estas, mesmo sendo consideradas pelos produtores como populações diferentes ("casco-fino caciquense" e "casco-grosso caciquense") não divergiram geneticamente. Estes dados de certa forma concordam com os resultados encontrados por Bisognin \& Marchezan (1988). Estes autores observaram restrita diversidade genética em dez populações de porongo coletadas na região de Santa Maria, estas populações se diferenciaram quanto a percentagem de frutos industriallizáveis.

A população proveniente de Jaguarí mostrou divergência entre as populações encontrados em Santa Maria e pelo método de Tocher foi colocada no segundo grupo ("casco-grosso precoce").

Os dados também foram submetidos ao método hierárquico do vizinho mais próximo (Single Linkage Method) que nos apresentou resultados concordantes com o método de Tocher, o método do vizinho mais próximo nos proporcionou uma ilustração gráfica da divergência genética das populações estudadas através de um 
dendograma dos grupos obtidos (Figura 1).

\section{Descritores qualitativos}

Os descritores qualitativos mostraram-se eficientes para a diferenciação das três populações ao contrário das obtidas pelas análises de agrupamento.

A tabela 1 apresenta a caracterização das populações a partir destes descritores. Assim na população denominada de "casco-fino caciquense" foi observado com relação ao descritor (A) as formas tanto agudas como obtusas e para o descritor (B) apenas a forma plana e para (CF) o caráter fistuloso. Na população "Cascogrosso caciquense" foi observado para (A) apenas o caráter agudo, já em (B) a forma convexa e em (CF) também o caráter fistuloso foi observado e na população "Casco-grosso Precoce" em (A) encontramos o caráter agudo, $(\mathrm{B})$ a forma plana e em $(\mathrm{C})$ o caráter cheio.

Em relação ao formato do fruto (FF) sugerimos a seguinte nomenclatura : achatado/piriforme (em formato de pêra), octoforme (em formato do número oito), gotiforme (em formato de gota), halteriforme (em formato de hálteres), globular/piriforme e eliptical/pririforme (em forma de elipse). Estas formas estão ilustradas na Figura 2.

Em relação ao formato do fruto foi observado grande variabilidade na população "Casco-fino caciquense" proveniente do município de Cacequí (RS), porém coletada em Santa Maria. Foram encontrados os seguintes formatos conforme a nomenclatura proposta : Achatado/Piriforme, Octoforme, Gotiforme e Halteriforme. Entretanto na variedade casco-grosso caciquense também proveniente do município de Cacequí e coletada em Santa Maria foi encontrado os seguintes formatos: Globular/Piriforme e Eliptical/Piriforme e por último a variedade Casco-grosso precoce coletada em jaguarí mostrou pouca variabilidade em relação ao formato do fruto, sendo verificado apenas o formato Achatado/Piriforme (Figura 2). 


\section{REFERÊNCIAS BIBLIOGRÁFICAS}

BISOGNIN, D. A .; AUDE, M. I. S.; MARCHEZAN, E. Densidade de semeadura e produtividade do porongo. Ciência Rural, Santa Maria, v. 22(1) p. 15-19, 1992.

BISOGNIN, D. A .; STORCK L. Estimativa dos componentes de variância e herdabilidade para formato de fruto em porongo Lagenaria siceraria (Mol.) Standl. Ciência Rural, v. 30, n.4, p.593597, 2000.

BISOGNIN, D. A., ESTEFANEL, V. Determinação do tamanho de parcela na cultura do porongo. Ciência Rural, v.18(3-4):197$200,1988$.

CRUZ, C. D., REGAZZI. A.J. Modelos biométricos aplicados ao melhoramento genético. Viçosa. MG: Editora UFV, 1994. 394 p.

ESQUINAS-ALCAZAR, J. T., GULICK, P. J. Genetic resources of cucurbitaceae. Rome: IBPGR secretariat, 1983, 101 p.

HOWES, C. Guidelines for developing descriptors lists. Plant Genetic Resources Newsletter, n. 45. P. 26-32, 1981.

RAO, C.R. Advanced statistical methods in biometric research. New York: Hafner, 1952. 390 p. 
Tab. 1. Descritores qualitativos dos frutos de L.siceraria utilizados para a caracterização das populações $(A=$ ápice, $B=$ base e $C F=$ cavidade da região proximal ao pedúnculo do fruto).

\begin{tabular}{cccc} 
Casco-grosso & $\begin{array}{c}\text { Casco-fino } \\
\text { caciquense }\end{array}$ & $\begin{array}{c}\text { Casco-grosso } \\
\text { precoce }\end{array}$ \\
\hline A & agudo & $\begin{array}{c}\text { agudo ou } \\
\text { obtuso }\end{array}$ & agudo \\
B & convexa & plana & plana \\
CF & fistuloso & fistuloso & cheio \\
\hline
\end{tabular}

Fig.1. Dendograma da divergência genética das populações de porongo de Santa Maria e Jaguarí (RS) pelo método do vizinho mais próximo (Single linkage method). Populações : 1 - "Casco-fino caciquense", 2 - "Casco-grosso caciquense" e 3 - "Casco-grosso precoce".

3

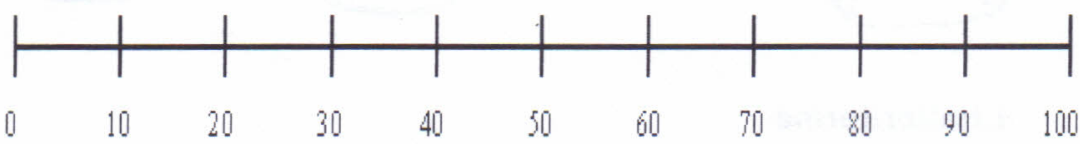




$$
\begin{aligned}
& 8303 \\
& 8003 \\
& 8083 \\
& 883
\end{aligned}
$$


5. Globular/Piriforme
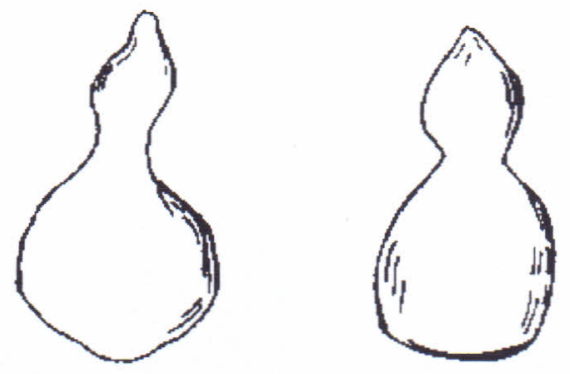

\section{Eliptical/Piriforme}

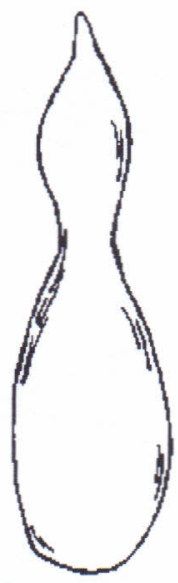


André LuÍs Lopes da Silva Acadêmico do Curso de Esp. em Biologia Centro de Ciências Naturais e Exatas Renato Aquino Záchia Mestre, prof. de Botânia Sistemática Centro de Ciências Naturais e Exatas Dilson Antônio Bisognin $\mathrm{PhD}$ em Genética e Melhoramento de Plantas Centro de Ciências Rurais Jean Carlos Budke Acadêmico do Curso de Ciências Biológicas Centro de Ciências Naturais e Exatas Universidade Federal de Santa Maria Santa Maria, RS - Brasil 